\title{
Interface Structure and Transport of Complex Oxide Junctions
}

B. B. Nelson-Cheeseman ${ }^{1}$, F. Wong ${ }^{1}$, R. V. Chopdekar ${ }^{2,1}$, M. Chi ${ }^{3,4}$, E. Arenholz ${ }^{5}$ N. D. Browning $^{3,4}$, and Y. Suzuki ${ }^{1}$.

${ }^{1}$ Dept. of Materials Science and Engineering, University of California, Berkeley, CA 94720.

${ }^{2}$ School of Applied and Engineering Physics, Cornell University, Ithaca, NY 14853.

${ }^{3}$ Dept. of Chemical Engineering and Materials Science, University of California, Davis, California 95616

${ }^{4}$ Materials Science and Technology Division, Lawrence Livermore National Laboratory, Livermore, California 94550

${ }^{5}$ Advanced Light Source, Lawrence Berkeley National Laboratory, Berkeley, CA 94720

\begin{abstract}
The interface structure and magnetism of hybrid magnetic tunnel junction-spin filter devices have been investigated and correlated with their transport properties. Magnetic tunnel junctions made of a spinel $\mathrm{NiMn}_{2} \mathrm{O}_{4}$ tunnel barrier sandwiched by theoretically predicted halfmetallic electrodes, perovskite $\mathrm{La}_{0.7} \mathrm{Sr}_{0.3} \mathrm{MnO}_{3}$ and spinel $\mathrm{Fe}_{3} \mathrm{O}_{4}$, exhibit very high crystalline quality as observed by transmission electron microscopy. Structurally abrupt interfaces allow for the distinct magnetic switching of the electrodes as well as large junction magnetoresistance. The change in the magnetic anisotropy observed at the spinel-spinel interface is indicative of a thin interdiffused magnetically soft interfacial layer. The strong exchange coupling at this interface allows for low background magnetoresistance, and a spin-filter effect with when the barrier is ferrimagnetic.

Materials: $\mathrm{Fe}_{3} \mathrm{O}_{4}, \mathrm{La}_{0.7} \mathrm{Sr}_{0.3} \mathrm{MnO}_{3}, \mathrm{NiMn}_{2} \mathrm{O}_{4}$
\end{abstract}


Interfaces play a crucial role in determining electrical transport across magnetic junction devices. Without considering the effects of the electrode-barrier interfaces, spin dependent transport behavior in magnetic junctions cannot be fully explained and understood. For example, the simple Julliere model ${ }^{1}$ of a magnetic tunnel junction (MTJ) where the conductance depends on only the relative bulk spin polarization of the electrodes does not adequately describe real MTJ behavior. It is now largely acknowledged that the electrode-barrier interfacial electronic structure needs to be taken into account to accurately describe MTJ experiments. ${ }^{2}$ Recently, magnon excitations at interfaces ${ }^{3}$ and bonding effects at the electrode/barrier interface ${ }^{4}$ have also been identified as factors affecting junction transport.

Our recent work on magnetic junctions composed of perovskite structure $\mathrm{La}_{0.7} \mathrm{Sr}_{0.3} \mathrm{MnO}_{3}$ (LSMO) and spinel structure $\mathrm{Fe}_{3} \mathrm{O}_{4}$ electrodes with spinel structure $\mathrm{NiMn}_{2} \mathrm{O}_{4}$ (NMO) barrier layers have shown that even within one junction, the transport can be dominated by the electrode-barrier interfaces or the bulk properties of the barrier layer itself depending on whether NMO is paramagnetic or ferrimagnetic, respectively. ${ }^{5}$ These two different conduction mechanisms directly highlight the relative contributions of the barrier layer itself in comparison to electrode-barrier interfaces to the spin-dependent transport. More specifically, above the $T_{C}$ of the NMO barrier, when the barrier layer is paramagnetic, the two different electrode-barrier interfaces dominate the magnetotransport behavior, resulting in an asymmetric bias dependence of the junction magnetoresistance (JMR) and inelastic tunneling spectra (IETS). Below the $\mathrm{T}_{\mathrm{C}}$ of the NMO barrier, the properties of the barrier dominate the magnetotransport behavior over that of electrode-barrier effects, resulting in a transition to a symmetric bias dependence of the JMR and IETS. ${ }^{5}$

Our discovery of the coexistence of magnetic tunneling behavior when the NMO is 
paramagnetic and spin filtering behavior when the NMO is ferrimagnetic suggests new routes in the design of magnetic devices where the transport can be tuned by the barrier layer. Both tunneling and spin filtering behavior, in different temperature regimes, are possible because of there is no magnetic coupling at the non-isostructural perovskite-spinel LSMO/NMO interface, but strong magnetic coupling at the isostructural spinel-spinel $\mathrm{NMO} / \mathrm{Fe}_{3} \mathrm{O}_{4}$ interface. This has been verified by element specific X-ray magnetic circular dichroism interface studies. ${ }^{5,6}$ However, the atomic structure of the LSMO/NMO perovskite-spinel interface and $\mathrm{NMO} / \mathrm{Fe}_{3} \mathrm{O}_{4}$ spinel-spinel interface must be explored in an effort to explain the magnetic interactions at these interfaces. The structure and magnetism at the interfaces must then be correlated with the transport behavior in these half-metallic oxide-based junctions.

In this paper, we correlate the interface structure of these hybrid MTJ-spin filter devices with the magnetotransport. With transmission electron microscopy (TEM) and scanning TEM (STEM) studies, we show the successful deposition of highly crystalline abrupt perovskite-spinel heterointerfaces. In crystalline $\mathrm{LSMO} / \mathrm{NMO} / \mathrm{Fe}_{3} \mathrm{O}_{4}$ heterostructures, the JMR is as high as $-30 \%$ and the magnetic switching is sharp and distinct, indicating that the electrodes are not magnetically coupled to each other. We show that the change in the magnetic anisotropy at the $\mathrm{NMO} / \mathrm{Fe}_{3} \mathrm{O}_{4}$ spinel interface supports the presence of a magnetically soft thin interdiffused interface layer of $(\mathrm{Fe}, \mathrm{Mn}, \mathrm{Ni})_{3} \mathrm{O}_{4}$, whose exchange coupling to the $\mathrm{Fe}_{3} \mathrm{O}_{4}$ electrode likely accounts for the low background magnetoresistance seen in these junctions, and the successful spin filtering when the barrier layer is ferrimagnetic.

For this study, MTJs of $\mathrm{LSMO} / \mathrm{NMO} / \mathrm{Fe}_{3} \mathrm{O}_{4}$ and $\mathrm{NMO}$ single layer films were synthesized. $\mathrm{Fe}_{3} \mathrm{O}_{4}$ and LSMO were chosen as electrode materials as they have theoretically and experimentally been shown to be half-metallic. ${ }^{7,8,9}$ Since isostructural barrier layers have proven 
to greatly increase the JMR values for $\mathrm{Fe}_{3} \mathrm{O}_{4}$-based MTJs, ${ }^{10}$ the ferrimagnetic spinel, $\mathrm{NiMn}_{2} \mathrm{O}_{4}$, was selected. The trilayers of $\mathrm{LSMO} / \mathrm{NMO} / \mathrm{Fe}_{3} \mathrm{O}_{4}$ were grown on (110)-oriented single crystal $\mathrm{SrTiO}_{3}$ (STO) substrates by pulsed laser deposition with a KrF excimer laser (248 nm) operating at $10 \mathrm{~Hz}$ with an energy density of approximately $1.5 \mathrm{~J} / \mathrm{cm}^{3}$. Both (110)-oriented LSMO and $\mathrm{Fe}_{3} \mathrm{O}_{4}$ films have strong in-plane uniaxial anisotropy, which is optimal for magnetic switching, along the [001] easy direction. Recent work on the manganites also suggests that the magnetism of (110) planes is more robust than that of (001) planes. ${ }^{11}$ The LSMO perovskite layer was deposited first at $700^{\circ} \mathrm{C}$ in 320 mtorr of $\mathrm{O}_{2}$. The $\mathrm{NMO}$ spinel layer was grown next at $550^{\circ} \mathrm{C}$ in $10 \mathrm{mT}$ Torr of a $99 \% \mathrm{~N}_{2} / 1 \% \mathrm{O}_{2}$ gaseous mixture. Single NMO films prepared under these conditions exhibit a $\mathrm{T}_{\mathrm{C}}$ of about $60 \mathrm{~K}$, a large coercive field of $1.8 \mathrm{~T}$ at $30 \mathrm{~K}$, and a magnetization of 0.8 $\mu \mathrm{B}$ /formula unit. Finally, the $\mathrm{Fe}_{3} \mathrm{O}_{4}$ spinel layer was synthesized at $400^{\circ} \mathrm{C}$ in vacuum. The bulk lattice parameters of the STO and LSMO perovskites are $3.905 \AA$ and $3.873 \AA$, respectively. The bulk lattice parameters of the $\mathrm{NMO}$ and $\mathrm{Fe}_{3} \mathrm{O}_{4}$ spinels are roughly twice that of the perovskites and are $8.379 \AA$ and $8.397 \AA$, respectively. This 2-to-1 perovskite-spinel unit cell stacking allows for near epitaxial growth of perovskite-spinel heterostructures, although a large lattice mismatch of almost $8 \%$ exists between the two structures. The junctions were composed of electrodes layers of 40-50 nm thick and NMO barrier thicknesses of 2.0, 3.0, and $4.5 \mathrm{~nm}$.

The crystallinity and epitaxy of the individual layers were investigated by high resolution X-ray diffraction on an X'Pert Pro MRD and cross-sectional TEM and STEM using a FEI F20 UT Tecnai microscope at the National Center for Electron Microscopy, Lawrence Berkeley National Laboratory (LBNL). Cross-sectional TEM was also used to study the interface structure in the trilayer heterostructure. Magnetization of the films was studied by a Quantum Design superconducting quantum interference device (SQUID) magnetometer. The magnetism at the 
interfaces was also investigated by element specific X-ray magnetic circular dichroism (XMCD) using total electron yield detection at beamlines 4.0.2 and 6.3.1 at the Advanced Light Source, LBNL. As the mean probe depth of these techniques is approximately $5 \mathrm{~nm}$, the bottom LSMO/NMO interface was investigated using a STO(110)/LSMO(40nm)/NMO(5nm) sample, while the top $\mathrm{NMO} / \mathrm{Fe}_{3} \mathrm{O}_{4}$ interface was investigated using a $\mathrm{STO}(110) / \mathrm{LSMO}(40 \mathrm{~nm}) / \mathrm{NMO}(5 \mathrm{~nm}) / \mathrm{Fe}_{3} \mathrm{O}_{4}(5 \mathrm{~nm})$ sample. The $\mathrm{Fe}_{3} \mathrm{O}_{4}$ magnetism away from the $\mathrm{NMO} / \mathrm{Fe}_{3} \mathrm{O}_{4}$ interface was also investigated with a $\mathrm{STO}(110) / \mathrm{LSMO}(40 \mathrm{~nm}) / \mathrm{NMO}(5 \mathrm{~nm}) / \mathrm{Fe}_{3} \mathrm{O}_{4}(8 \mathrm{~nm})$ sample, in which the top $\mathrm{Fe}_{3} \mathrm{O}_{4}$ layer was sufficiently thick so that the $\mathrm{NMO} / \mathrm{Fe}_{3} \mathrm{O}_{4}$ interface does not contribute to the electron yield signal.

The MTJ structures were fabricated by conventional UV contact alignment photolithography and Ar ion milling. Magnetotransport measurements, including resistance versus applied magnetic field and current versus voltage, were taken between $5 \mathrm{~K}$ and $400 \mathrm{~K}$ and up to 8 kOe with a modified Quantum Design Physical Property Measurement System (PPMS). The magnetic field was applied along the [001]-in-plane magnetically easy direction of the two magnetic electrodes. The JMR were calculated in accordance with Julliere's model by the following equation: $\left[\Delta R / R_{P}\right] * 100$ where $\Delta \mathrm{R}=\mathrm{R}_{A P}-\mathrm{R}_{P}$. The reference (parallel magnetization) resistance was taken as the resistance at $8 \mathrm{kOe}$ in the high junction resistance state.

$\mathrm{X}$-ray diffraction taken of the trilayer heterostructures indicates excellent crystallinity and epitaxy. Scans taken in the $2 \theta-\theta$ geometry show only $\{110\}$-oriented peaks for the $\mathrm{Fe}_{3} \mathrm{O}_{4}, \mathrm{NMO}$ and LSMO layers grown on (110) STO substrates, thus indicating out-of-plane epitaxy. Phi scans of the heterostructures also demonstrate in-plane registry with two-fold symmetry of the $\{001\}$ oriented peaks. 
Cross sectional TEM elucidates both the microstructure of the perovskite and spinel as well as the coherence of the perovskite-spinel (LSMO/NMO) interface. The phase-contrast TEM lattice image in Figure 1(a) demonstrates that it is possible to obtain abrupt interfaces between the LSMO perovskite and $\mathrm{NMO} / \mathrm{Fe}_{3} \mathrm{O}_{4}$ spinel layers in the magnetic junctions. Figure 1 (a) was taken of the trilayer structure along the [001] zone axis and shows highly crystalline LSMO, an abrupt and coherent spinel-perovskite interface, and spinel layers with high crystallinity. The spinel-spinel interface cannot be identified by TEM or STEM due to the similarities in both atomic number and crystal structure of $\mathrm{NMO}$ and $\mathrm{Fe}_{3} \mathrm{O}_{4}$. The epitaxy of the trilayer is confirmed by the Fast Fourier Transform on the TEM image, shown in Figure 1(b), where the double spots are a signature of the lattice mismatch of LSMO and spinel layers, and demonstrate both in-plane and out-of-plane crystalline registry of the spinel with the perovskite template. Despite the relatively large lattice mismatch between the perovskite and spinel films, the spinel layers grow coherently on the LSMO with crystalline registry and good crystalline quality. However, the large lattice mismatch between the perovskite and spinel structures inevitably creates defects at the perovskite-spinel interface as well as in the spinel layers themselves. The high-resolution STEM image of the $\mathrm{Fe}_{3} \mathrm{O}_{4}$ film in Figure 1(c) shows that we obtain very high-quality crystallinity of the $\mathrm{Fe}_{3} \mathrm{O}_{4}$ on a local atomic level, despite extended defects. Although the spinel films are not perfectly epitaxial, a combination of low-angle grain boundaries, anti-phase boundaries, and dislocations act as mechanisms for lattice relaxation that allow the spinel films to maintain good structural registry with the perovskite underlayer. While in general defects in heterostructures may be seen as undesirable, defects observed in the spinel $\mathrm{Fe}_{3} \mathrm{O}_{4}$ are crucial in the ability to grow relatively thick crystalline spinel films on highly mismatched perovskite underlayers. In addition, as seen from both SQUID magnetometry and XMCD measurements, 
these defects do not significantly degrade the magnetic properties of the $\mathrm{Fe}_{3} \mathrm{O}_{4}$ as compared to bulk, and they prevent the coupling of the LSMO to the spinel NMO and $\mathrm{Fe}_{3} \mathrm{O}_{4}$ at the perovskite/spinel interface. ${ }^{5}$

Having established the structural integrity of the trilayer heterostructures, the magnetic order near each interface was probed by surface sensitive XMCD using total electron yield detection in specially prepared samples. ${ }^{5,6}$ Element specific hysteresis loops can be obtained by choosing specific X-ray energies corresponding to the $\mathrm{Mn}, \mathrm{Ni}$ and $\mathrm{Fe} \mathrm{L}_{2,3}$ absorption edges. At the LSMO/NMO interface, the magnetism was probed only at the $\mathrm{Mn}_{3,2}$ absorption edges because the $\mathrm{Ni}_{2,3}$ absorption edges overlap with the $\mathrm{La} \mathrm{M}_{4,5}$ absorption edges of LSMO. While Mn is found in both NMO and LSMO, the differences in valence and site symmetry of the Mn ions in the spinel and perovskite structures allows for the differentiation and identification of the Mn in each layer. Mn XMCD hysteresis loops taken at two different energies, $640.0 \mathrm{eV}$ and $642.5 \mathrm{eV}$, in the Mn XMCD spectrum exhibit magnetically hard and magnetically soft behavior, respectively, at $55 \mathrm{~K}$ as shown in Figure 2. These two energies correspond to $\mathrm{Mn}^{2+}$ in the NMO layer and $\mathrm{Mn}^{3+/ 4+}$ in the LSMO layer, respectively. ${ }^{5}$ Since the two loops are independent of each other, there appears to be no noticeable coupling of magnetic ions at the LSMO/NMO interface even when the NMO is ferrimagnetic. This magnetic decoupling of the adjacent magnetic layers is necessary to achieve the spin-filter effect observed in these junctions. ${ }^{5}$

At the $\mathrm{NMO} / \mathrm{Fe}_{3} \mathrm{O}_{4}$ interface, both $\mathrm{Ni}$ and $\mathrm{Mn}$ exhibit long range magnetic order at room temperature and their hysteresis loops coincide with those of $\mathrm{Fe}^{6}{ }^{6}$ Although the normalized XMCD hysteresis loops from the trilayer sample are identical for $\mathrm{Ni}, \mathrm{Mn}$ and $\mathrm{Fe}$ at all temperatures, the shape of the hysteresis loops changes distinctly below $60 \mathrm{~K}$, exhibiting magnetically harder hysteresis loops once the NMO layer becomes ferrimagnetic. These 
coincident loops are strong evidence for magnetic coupling at the $\mathrm{NMO} / \mathrm{Fe}_{3} \mathrm{O}_{4}$ interface, causing the $\mathrm{NMO} / \mathrm{Fe}_{3} \mathrm{O}_{4}$ layers to act as a single magnetic stack, rather than two independent layers. We have also recently found that at the $\mathrm{NMO} / \mathrm{Fe}_{3} \mathrm{O}_{4}$ interface there is a thin interdiffused region of (Fe,Mn,Ni) ${ }_{3} \mathrm{O}_{4}$ leading to $\mathrm{Mn}$ and $\mathrm{Ni}$ magnetic properties similar to $\mathrm{MnFe}_{2} \mathrm{O}_{4}$ and $\mathrm{NiFe}_{2} \mathrm{O}_{4}$ by XMCD and X-ray absorption spectroscopy. ${ }^{6}$

The presence or absence of magnetic ordering of $\mathrm{NiMn}_{2} \mathrm{O}_{4}$ dictates the magnetic coupling of the interfacial sublayer in the spinel stack. A detailed analysis of the magnetic properties of the sublayer region by $\mathrm{XMCD}$ indicates that the interdiffused $(\mathrm{Fe}, \mathrm{Mn}, \mathrm{Ni})_{3} \mathrm{O}_{4}$ layer is magnetically softer than the $\mathrm{NMO}$ and $\mathrm{Fe}_{3} \mathrm{O}_{4}$ layers. Whereas the magnetically hard nature of the NMO layer was evidenced while investigating the LSMO/NMO interface and is shown in Figure 2, the bulk $\mathrm{Fe}_{3} \mathrm{O}_{4}$ layer also has a larger coercive field than the interdiffused sublayer region, as shown in Figure 3. Therefore, at the $\mathrm{NiMn}_{2} \mathrm{O}_{4} /(\mathrm{Fe}, \mathrm{Mn}, \mathrm{Ni})_{3} \mathrm{O}_{4} / \mathrm{Fe}_{3} \mathrm{O}_{4}$ interface, the magnetically soft interdiffused sublayer couples to the $\mathrm{Fe}_{3} \mathrm{O}_{4}$ and $\mathrm{NMO}$ layers. When the NMO layer is paramagnetic, the sublayer magnetic moments magnetically switch with the interfacial $\mathrm{Fe}_{3} \mathrm{O}_{4}$ moments [Figure 4(a)]. When the NMO is ferrimagnetic, the sublayer and $\mathrm{Fe}_{3} \mathrm{O}_{4}$ moments switch with the interfacial NMO moments, resulting in an abrupt increase in coercive field below the NMO $\mathrm{T}_{\mathrm{C}}[$ Figure 4(b)].

Hysteresis loops taken of the $\mathrm{NMO} / \mathrm{Fe}_{3} \mathrm{O}_{4}$ interface in a trilayer sample also demonstrate that the in-plane [11-0] direction is in fact magnetically easier for the interfacial $\mathrm{Fe}, \mathrm{Mn}$ and $\mathrm{Ni}$ than the in-plane [001] direction both above and below the Curie temperature of the NMO barrier layer. This anisotropy is in contrast with the in-plane [001] easy direction exhibited by both the $\mathrm{LSMO}$ and $\mathrm{Fe}_{3} \mathrm{O}_{4}$ electrodes grown on $\mathrm{STO}(110)$ substrates. This observation provides further evidence that a $\left(\mathrm{Fe}, \mathrm{Mn}, \mathrm{Ni}_{3} \mathrm{O}_{4}\right.$ interfacial sublayer is present which exhibits properties 
similar to $\mathrm{MnFe}_{2} \mathrm{O}_{4}$ and $\mathrm{NiFe}_{2} \mathrm{O}_{4}{ }^{12}$

The transport properties of the MTJs with an abrupt LSMO/NMO interface and an interdiffused $\mathrm{NMO} / \mathrm{Fe}_{3} \mathrm{O}_{4}$ interface exhibit square junction magnetoresistance (JMR) loops with flat background magnetoresistance (MR) at high magnetic fields. As shown in Figure 5(a), transitions in the magnetization hysteresis loops coincide well with large and abrupt transitions in the JMR. The low resistance state occurs when the two magnetic electrodes are magnetized antiparallel to one another resulting in negative JMR values. This negative JMR is due to the opposite spin polarizations of the LSMO and $\mathrm{Fe}_{3} \mathrm{O}_{4}$ electrodes, which are majority and minority spin polarized, respectively. ${ }^{10}$ At high magnetic fields, the background magnetoresistance (MR) as a fraction of the maximum JMR for these junctions, shown in Figure 5(b), is significantly lower than that previously seen in similar $\mathrm{LSMO}-\mathrm{Fe}_{3} \mathrm{O}_{4}$ junctions with other spinel barrier layers. These junctions exhibited background MR values two to four times larger when barrier layers of $\mathrm{CoCr}_{2} \mathrm{O}_{4}, \mathrm{MgTi}_{2} \mathrm{O}_{4}$ and $\mathrm{FeGa}_{2} \mathrm{O}_{4}$ were used. ${ }^{10,12}$

The interface structure and magnetic behavior of the NMO barrier layer can be correlated to the junction transport. First, the abrupt switching of the JMR, even when the NMO is magnetic, indicates that the use of a magnetic barrier layer does not preclude the accessibility of distinct parallel and antiparallel spin polarized states at the two electrode-barrier interfaces. The structurally distinct perovskite-spinel interface seen in the cross-sectional TEM likely contributes to the abrupt switching of the electrodes near the electrode-barrier interface by decreasing any electrode-electrode or electrode-barrier orange-peel coupling. Furthermore, the misfit dislocations present at the spinel-perovskite interface seem to eliminate exchange coupling between the magnetic layers across the non-isostructural interface, thereby decoupling the perovskite and spinel layers, allowing for an antiparallel magnetization configuration between 
the electrodes.

Moreover, the relatively low background magnetoresistance of these junctions compared to other $\mathrm{LSMO} / \mathrm{Fe}_{3} \mathrm{O}_{4}$-based junctions may be associated with the properties of the thin interfacial $(\mathrm{Fe}, \mathrm{Mn}, \mathrm{Ni})_{3} \mathrm{O}_{4}$ layer at the $\mathrm{NMO} / \mathrm{Fe}_{3} \mathrm{O}_{4}$ interface and its interaction with the surrounding $\mathrm{Fe}_{3} \mathrm{O}_{4}$ and NMO. The low background MR indicates that the magnetic switching of spins at the $\mathrm{Fe}_{3} \mathrm{O}_{4}$ electrode interface is more abrupt as compared to previous $\mathrm{Fe}_{3} \mathrm{O}_{4}$-based MTJs.

In junctions that exhibit such low background $M R$, it is surprising that the magnetically easy direction of the interfacial spinel sublayer detected at the $\mathrm{NMO} / \mathrm{Fe}_{3} \mathrm{O}_{4}$ interface is not coincident with that of the $\mathrm{Fe}_{3} \mathrm{O}_{4}$ electrode above $60 \mathrm{~K}$, since such modulation of the interfacial magnetic anisotropy should contribute to misalignment of the spins at the electrode-barrier interface. However, the presence of this specific, predominately $\mathrm{MnFe}_{2} \mathrm{O}_{4}$-like spinel sublayer may in fact aid in the alignment of the $\mathrm{Fe}_{3} \mathrm{O}_{4}$ spins to the bulk of the $\mathrm{Fe}_{3} \mathrm{O}_{4}$ layer, resulting in lower background MR compared to other junctions, in the following way. When the field is applied in-plane along the [001]-direction, the magnetization of the $\mathrm{Fe}_{3} \mathrm{O}_{4}$ likely causes the magnetically soft interfacial sublayer spins to experience a large molecular field, resulting in strong exchange coupling across the interface. Such exchange coupling between magnetically soft and magnetically hard spinel ferrite thin films has been shown to be quite strong. ${ }^{14}$ Furthermore, the strength of the interaction is inversely proportional to the thickness of the soft ferrite layer, ${ }^{14}$ indicating that a magnetically soft ferrite sublayer on the order of $1-2 \mathrm{~nm}$ thick should easily couple to a magnetically harder ferrite layer greater than $40 \mathrm{~nm}$ in thickness. Therefore, when the bulk of the $\mathrm{Fe}_{3} \mathrm{O}_{4}$ switches, so does the interfacial sublayer, even if the field is not applied along the easy direction of the spinel electrode interfacial spins. This results in less background MR, as well as a greater JMR seen at each bulk electrode switching event. 
Unfortunately, verification of the exchange coupling of the sublayer region to the full $40 \mathrm{~nm}$ $\mathrm{Fe}_{3} \mathrm{O}_{4}$ top layer is difficult to verify in these heterostructures, as element-specific, surfacesensitive soft $\mathrm{x}$-ray techniques cannot access a $40 \mathrm{~nm}$-deep sublayer region, and the magnetization of the sublayer would be overwhelmed by the bulk $\mathrm{Fe}_{3} \mathrm{O}_{4}$ layer in bulk techniques.

In summary, we have investigated the structure and magnetic properties of hybrid MTJspin filter devices and how they affect the magnetotransport properties. The crystalline structure of the heterostructure causes the lack of magnetic coupling at the non-isostructural LSMO/NMO interface, and the strong magnetic coupling observed at the isostructural $\mathrm{NMO} / \mathrm{Fe}_{3} \mathrm{O}_{4}$ interface. In addition, the presence of a magnetically soft layer with a modified magnetic anisotropy at the isostructural $\mathrm{NMO} / \mathrm{Fe}_{3} \mathrm{O}_{4}$ interface strongly suggests the existence of a predominately $\mathrm{MnFe}_{2} \mathrm{O}_{4}{ }^{-}$ like interdiffused sublayer, whose exchange coupling to the $\mathrm{Fe}_{3} \mathrm{O}_{4}$ electrode likely accounts for the low background magnetoresistance seen in these junctions, and the successful spin filtering when the NMO barrier layer is ferrimagnetic Nonlinear junction transport observed both above and below the $\mathrm{T}_{C}$ of NMO indicates that the insulating NMO is an effective potential barrier both in its paramagnetic and ferrimagnetic states. This work demonstrates that introducing a magnetic barrier layer can produce novel effects in MTJ-type structures, thereby creating a new paradigm for the design of spin-based devices.

This work was supported in full by the Office of Basic Energy Sciences of the U.S. Department of Energy under Contract No. DE-AC02-05CH11231. Processing performed in the University of California-Berkeley Microlab. 
Figure 1 - Structural characterization of the junction heterostructure taken along the [001] zone axis. (a) High resolution TEM image of the perovskite (LSMO)-spinel (NMO/Fe3O4) interface, (b) Fast Fourier Transform of the TEM image, (c) STEM image taken of the $\mathrm{Fe}_{3} \mathrm{O}_{4}$. Schematics show arrangement of tetrahedral Fe (green), octahedral Fe (blue) and $\mathrm{O}$ (red) ions along atomic columns.

Figure 2 - XMCD of the LSMO/NMO interface. (a) Mn $\mathrm{L}_{2,3}$ XMCD spectra taken at 55K of the LSMO/NMO bilayer with the mean XMCD probe depth demonstrated on the sample schematic. XMCD hysteresis loops taken at (b) $640.0 \mathrm{eV}$ and (c) $642.5 \mathrm{eV}$.

Figure 3 - Room temperature in-plane Fe XMCD hysteresis loops taken along the [001] direction of the interdiffused $(\mathrm{Fe}, \mathrm{Mn}, \mathrm{Ni})_{3} \mathrm{O}_{4}$ sublayer at the $\mathrm{NMO} / \mathrm{Fe}_{3} \mathrm{O}_{4}$ interface (open circles) and of only the top $\mathrm{Fe}_{3} \mathrm{O}_{4}$ electrode in the trilayer heterostructure (closed circles). Sample schematics demonstrate the two samples probed in this study.

Figure 4 - Fe hysteresis loops taken at $30 \mathrm{~K}$ and $80 \mathrm{~K}$ along the [001] and [11-0] in-plane crystallographic directions for the trilayer sample shown.

Figure 5 - Junction transport as a function of applied magnetic field at 55K. (a) JMR and moment at low magnetic fields and (b) complete JMR curve including high magnetic fields. 
Figure 1
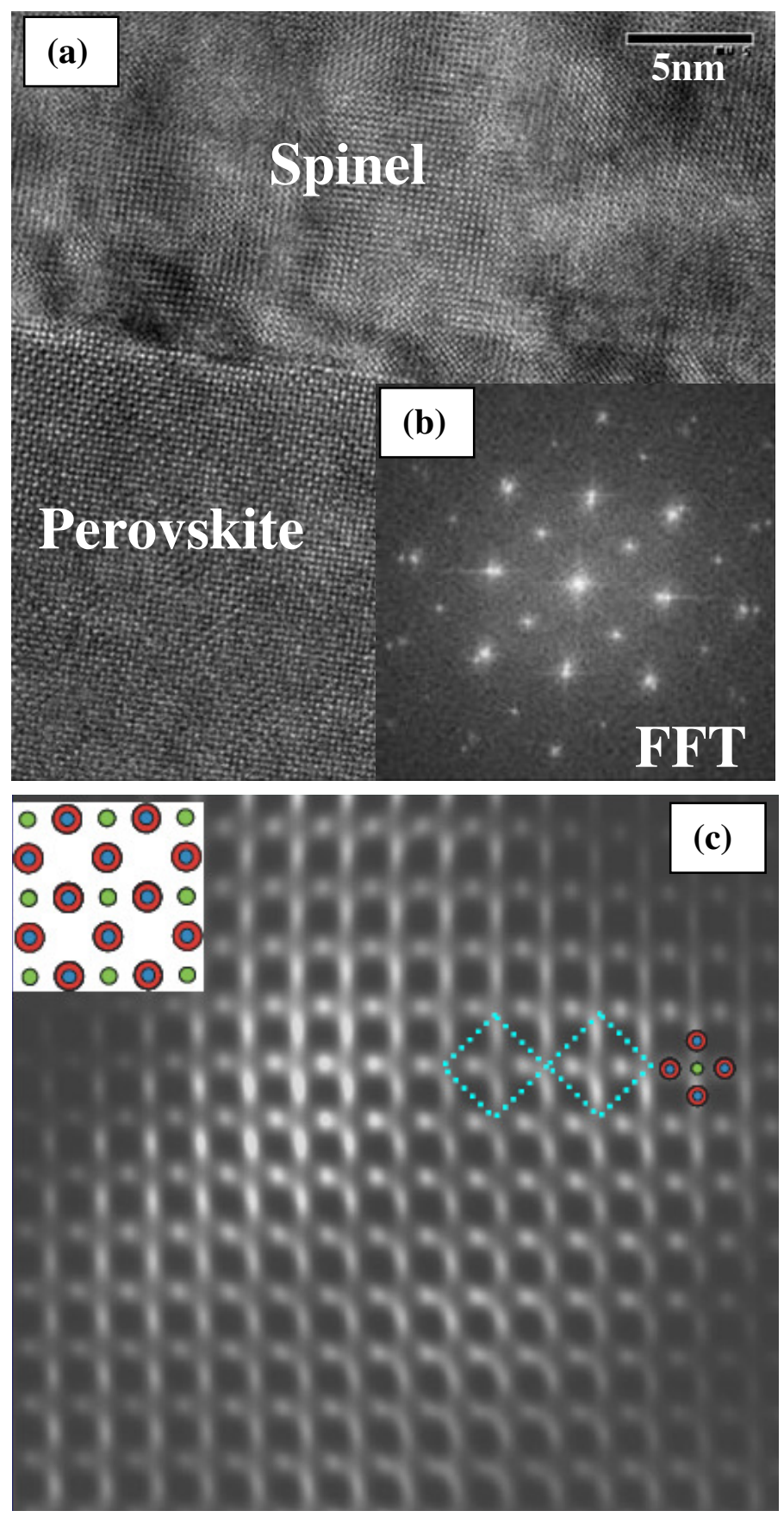
Figure 2

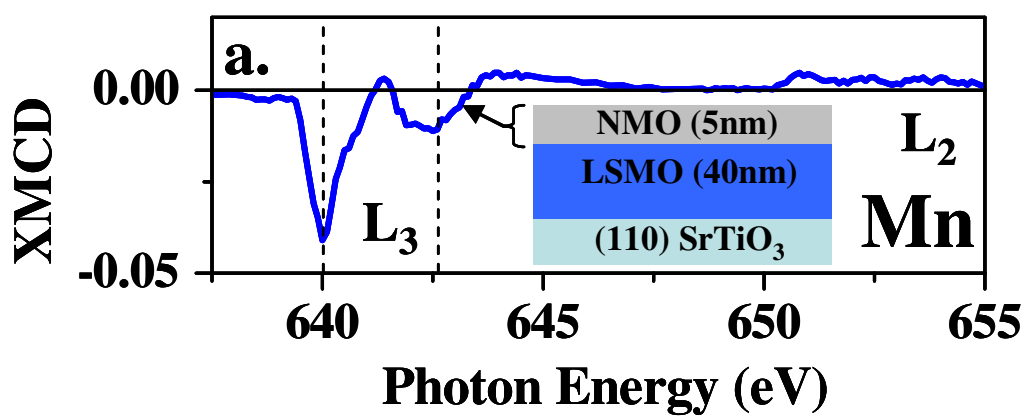

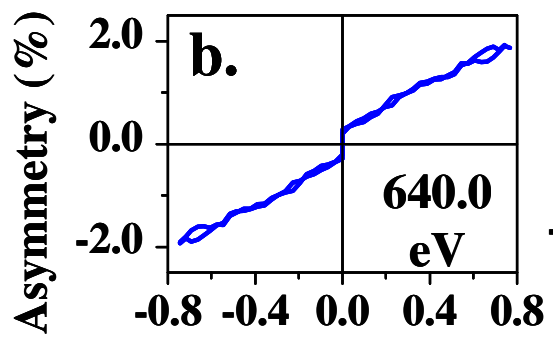

Applied Field (T)

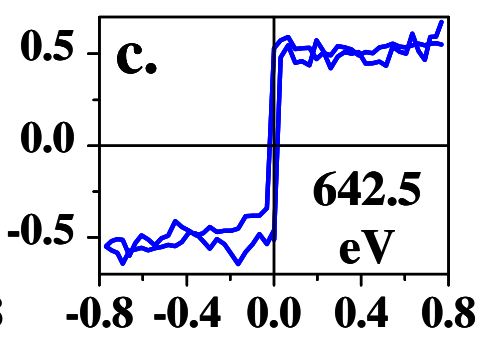

Applied Field (T) 
Figure 3

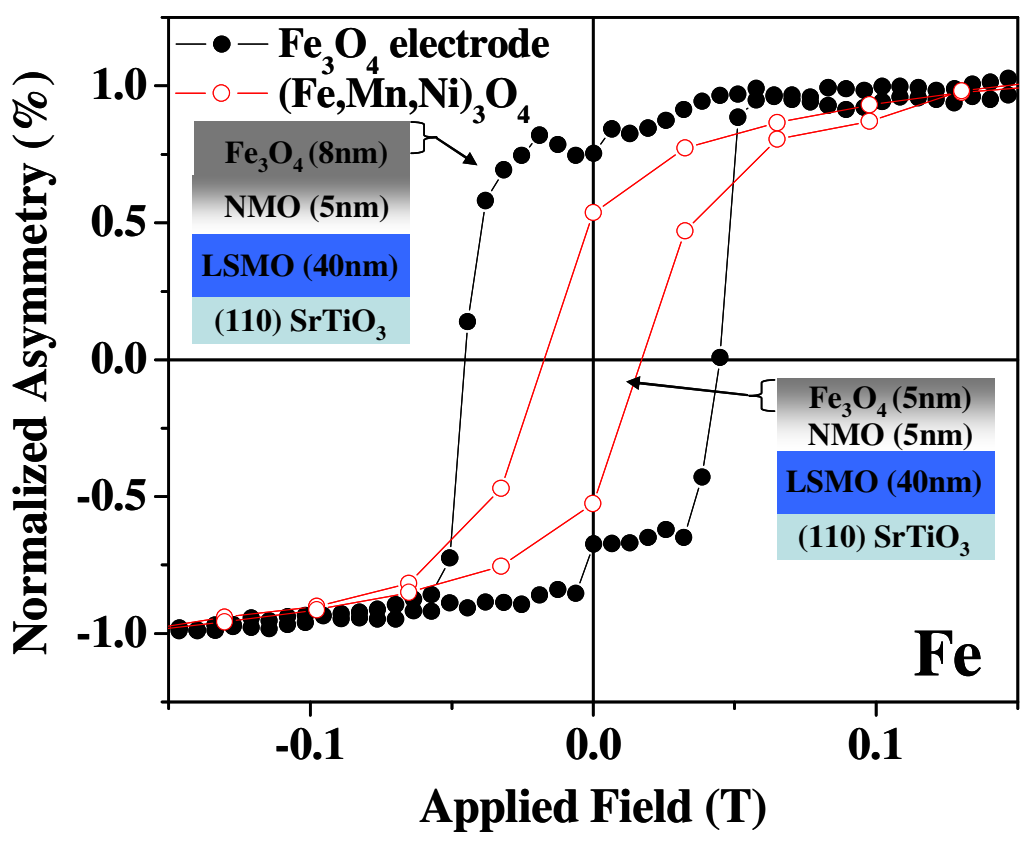


Figure 4
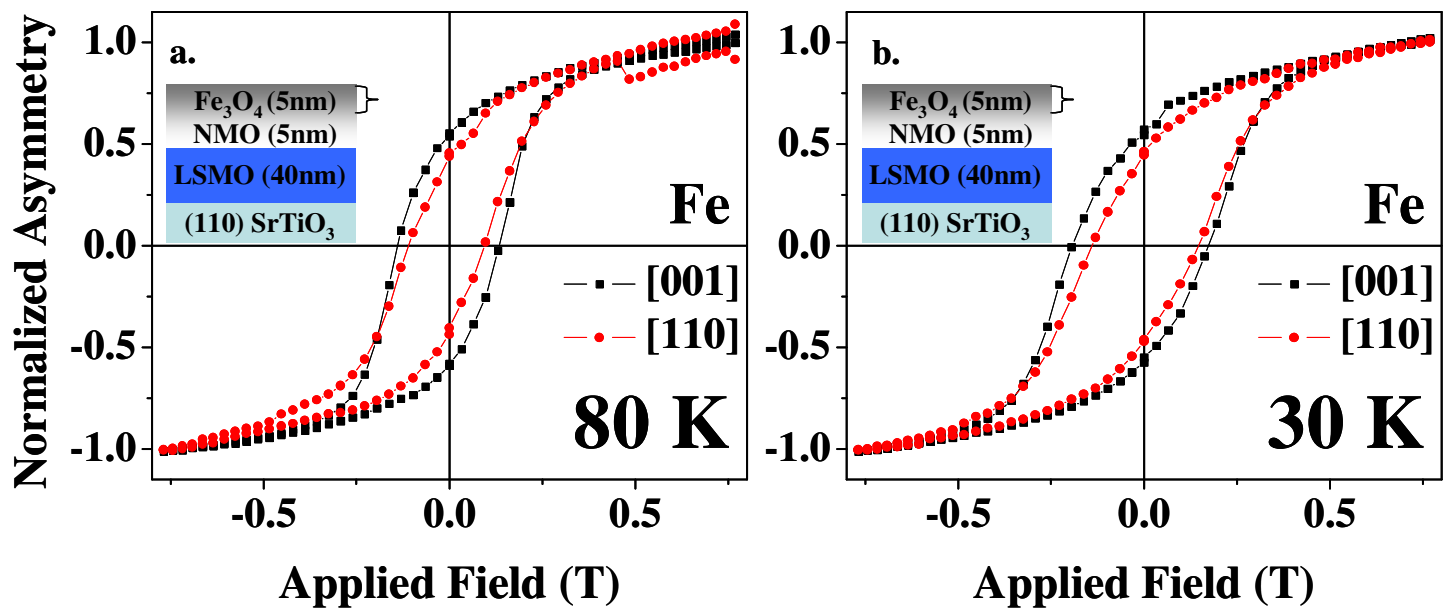
Figure 5
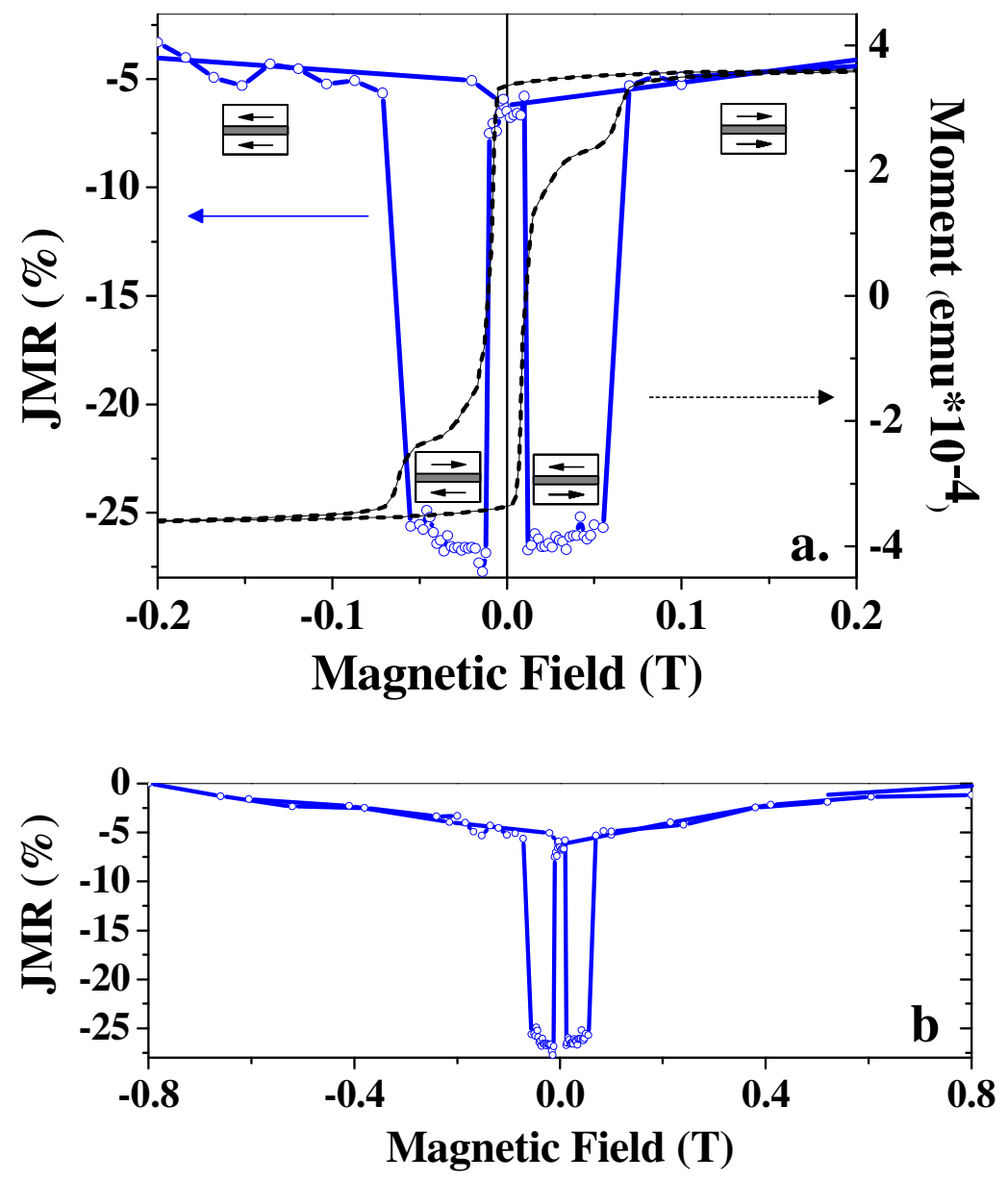


\section{REFERENCES:}

1. M. Julliere, Phys. Lett. 54A, 225 (1975).

2. G. T. Woods, R. J. Soulen,Jr., I. I. Mazin, B. Nadgorny, M. S. Osofsky, J. Sanders, H.

Srikanth, W. F. Egelhoff, R. Datla, Phys. Rev. B. 70, 054416 (2004).

3. J. S. Moodera, L. R. Kinder, T. M. Wong, and R. Meservey, Phys. Rev. Lett. 74, 3273 (1995).

4. W. H. Butler, X. -G. Zhang, T. C. Schulthess, J. M. MacLaren, Phys. Rev. B 63, 054416 (2001).

5. B. B. Nelson-Cheeseman, R. V. Chopdekar, L. M. B. Alldredge, J. S. Bettinger, E. Arenholz, and Y. Suzuki. Phys. Rev. B 76, 220410(R) (2007).

6. B. B. Nelson-Cheeseman, R. V. Chopdekar, J. S. Bettinger, E. Arenholz, and Y. Suzuki. J. Appl. Phys. (to be published).

7. A. Yanase and K. Siratori, J. Phys. Soc. Jpn. 53, 312 (1984).

8. Ze Zhang and Sashi Satpatahy, Phys. Rev. B 44, 13319 (1991).

9. W. E. Picket, D. J. Singh, J. Magn. Magn. Mater. 172, 237 (1997).

10. G. Hu and Y. Suzuki, Phys. Rev. Lett. 89, 276601 (2002).

11. I. C. Infante, F. Sánchez, J. Fontcuberta, M. Wojcik, E. Jedryka, S. Estradé, F. Peiró, J. Arbiol, V. Laukhin, and J. P. Espinós. Phys. Rev. B 76, 224415 (2007).

12. S. E. Harrison, C. J. Kriessman, and S. R. Pollack, Phys. Rev. 110, 844 (1958).

13. L. M. B.Alldredge, R. V. Chopdekar, B. Nelson-Cheeseman, and Y. Suzuki, Appl. Phys. Lett. 89, 182504 (2006).

14. Y. Suzuki, R. B. van Dover, E. M. Gyorgy, Julia M. Phillips, and R. J. Felder, Phys. Rev. B 53, 14016 (1996). 